அறேபிய புலம்பெயர் இலக்கியத்தில் தத்துவார்த்த உரையாடல்: கலீல் ஜிப்ரானின் கவிதைகளை துணையாகக் கொண்ட ஆய்வு

எம்.எச்.ஏ. முனாஸ் அ,*

அ அரபுத்துறை, இலங்கை தென்கிழக்குப் பல்கலைக்கழகம், ஒலுவில், இலங்கை.

\title{
Philosophical dialogue in Arabic diaspora literature with special reference to Kahlil Gibran's poems
}

\author{
MHA. Munas a, ${ }^{*}$ \\ a Department of Arabic, Faculty of Islamic Studies \& Arabic Language, South Eastern University, Sri Lanka. \\ *Corresponding author Email: munasmha@gmail.com \\ DOI: https://doi.org/10.54392/ijot2213
}

Received: 31-12-2021; Revised: 24-01-2022; Accepted: 29-01-2022; Published: 02-02-2022

\begin{abstract}
Philosophy regulates the functioning of human life. Good philosophies are the cross-cutting faces of human life. This theoretical thinking in Arabic literature dates back to the Jahiliyya period. Arab literary figures that, have appeared throughout history, have made significant contributions to the regulation of human life by conveying philosophical ideas in their literary works. These philosophical dialogues have the potential to elicit a positive response in solving the various social, economic and political issues of human life. In this manner, the Lebanese Arab diaspora poet Khalil Gibran is one of the foremost contributors to the Arabic literary world. The research tries to introduce Arabic diaspora literatue and poet Khalil Zibran and to express the theoretical and social ideas expressed in his poems. For this purpose, descriptive and analytical methods were used and studied. The poet Khali Gibran writes in these poems all his philosophical thoughts and social views, and speaks in it with the senses of nature and its details, arguing that nature is the cause of complete happiness and that the world is free from hypocrisy. This is what he describes as the characteristic of souls who strive to achieve their distant goal behind perfect human life away from the false world of materialism.
\end{abstract}

Keywords: Arabic language, Philosophical dialogue, Arabic diaspora literature, Kahlil Gibran, Poem

முன்னுரை

மனிதனுள் உணர்வு, சிந்தனை, அச்சம் தொடர்பாக எழும் மானுட எழுச்சியை மனித ஆக்கமாக எழுதும் பெரும் படைப்பே இலக்கியமாகும். மனிதன் தனது உணர்வுகளை வெளிப்படுத்த எழுத்துவகைசார்ந்த முறையை பின்பற்றுகின்றான். எழுத்தின் மூலம் விசாலமானதொரு துறையை தனது கருத்துக்களை வெளிப்படுத்த தேர்வு செய்கின்றன்றான். அதுவோ பின்னர் இலக்கியப் படைப்பாக பரிணாமமடைகின்றது. இங்கு இலக்கியத்திற்கும் மொழிக்குமிடையிலான உறவு விசாலப்பட்டு, பலமடைகிறது. மாத்திரமன்றி இலக்கியத்தினால் மொழி பாதுகாக்கப்பட்டு வருகின்றது. இவ்விலக்கியம் அழகியல், கற்பினை, கருத்தருவாக்கம் என்பன கலந்த, கருத்துச்செறிவுமிக்க கவிதை அல்லது உரை வடிவங்களில் அமைகின்றது. இதனால், இலக்கியமானது மொழியின் தன்மை, தலைப்பு, காலப்பகுதி, கலாசாரப்பின்னணி போன்றவற்றிற்க்கேற்ப பலவகையாகப் பிரிக்கப்படுவதைக் காணலாம்.

முன்னரைப்பார்க்கிலும் இலக்கியம் இருபதாம் நூற்றாண்டில் பாரிய வளர்ச்சியைக் கண்டுள்ளது. அதன் கிளைகள் விசாலித்து, வளர்ந்துள்ளன. 'இருபதாம் நூற்றாண்டில் ஏற்பட்ட சமூக மாற்றம் இக்கால இலக்கியங்களை அதற்கு முற்பட்ட கால இலக்கியங்களிலும் நின்று வேறுபடுத்துகின்றது. 
பிரித்தானியர் வருகையும், அவர்கள் பகுத்திய வர்த்தகப் பொருளாதார முறையும் காரணமாக நிலபிரபுத்துவ சமூக அமைப்பு சிதைவடைய, முதலாலித்துவ சமூக அமைப்பும் அதன் விளைவான நவீனமயமாதலும் தோன்றின. நிலபிரபுத்துவ வமூக அமைப்பிற் சமயமே இலக்கியத்தின் உள்ளடக்கமாக இருக்க சாதாரண மனிதனும் அவன் வாழ்க்கை நெருக்கடிக்களும் இலக்கியத்திற்கு அப்பால் இருந்தன எனலாம். முதலாளித்துவ வளர்ச்சியுடன் தனிமனித பிரச்சினைகள் முக்கியத்துவம் பெறத் தொடங்கவே, இந்நூற்றாண்டு இலக்கியம் அதன் வாழ்வைப் பொருளாகக் கொள்வது தவிர்க்க முடியாததாகி விட்டது. இலக்கியம் சமய சமயநெறியிலிருந்து சமூக நெறிக்கு மாறியது' (Maunaguru, Chirtalega \& Nuhman, 1979).

இந்த இலக்கியச் சூழலில், அறபு இலக்கியம் தனக்கான தனித்துவங்களைப் பேணி வரலாற்றில் வளர்ச்சியுற்றுள்ளதைக் காண முடிகின்றது. 'ஏனைய இலக்கியங்களுடன் ஒப்பிடுகையில் அறபு இலக்கியம் செழிப்புமிக்கதாகவே உள்ளது. அது மனிதனின் ஆரம்பம் முதல் அறேபிய நாகரிகம் சிதைவதைந்தது வரையான தொன்மையான இலக்கிய வரலாற்றினைக் கொண்டது. ஆரம்பத்தில் 'முழர்' வம்சத்தினரின் மொழியாகவிருந்த அறபு மொழி இஸ்லாத்தின் வருகையுடன் 'ஒற்றுமைப்பட் ஒரே சமூகத்தின்' மொழியாக மாறியது' (Alzayat, 2008). இதற்கு இஸ்லாமிய சமயமே காரணமாகும். இஸ்லாத்திற்காக தமது மொழியை, கருத்தாக்கங்களையெல்லாம் விட்டு, ஒரே மார்க்கத்தை, மொழியை, காலாசாரத்தை, நாகரீகத்தை எடுத்துக்கொண்டனர். இதனால், அறபு இலக்கிய மானுட வரலாற்றில், உயிர்ப்புடன் காரியமாற்றத் தொடங்கியது.

அறபு இலக்கியத்தின் விஷேட கூறாக கவிதை இலக்கியத்தைப் பார்க்க முடியும். பொதுவில் கவிதை இலக்கியமானது 'உண்மைக்கும், பொய்மைக்கும் இடையே ஏற்படும் போராட்டம், அதிகாரவர்க்கம், மக்கள் இவர்களுக்கு இடையேயான மோதல், தொழில்நுட்பம், வாழ்க்கையின் உயிர்த்துடிப்பு போன்றவற்றைப் பேசவும் பின்நிற்கவில்லை. இதனால் அவை மொழிபெயர்க்கப்பட்டன.' இந்தக் கவிதை இலக்கியப் பணிகளை அறபுக் கவிதைகளும் ஆற்றத்தவரவில்லை. அதேவேள, நவீன காலத்தின் பேசுபொருள்களுக்கேற்ப அறபுக் கவிதைகளும் செயற்படத் தொடங்கின. 'கவிதை என்பது அறபு மொழியின் சாராம்சம் என அறேபியர் தீவிரமாக நம்புகின்றனர். இது குடிமக்கள் மொழியியல் சொற்களைப் புரிந்துகொள்ள உதவுகிறது. 20 ஆம் நூற்றாண்டு வரை, இஸ்லாத்தின் வருகைக்கு முந்தைய கவிதைகள் பல்வேறு வடிவங்களில் நிலையான கட்டமைப்பைக் கொண்டிருந்தன. இது அறபு இலக்கியத்தை பெரிதும் பாதித்தது. 19 ஆம் நூற்றாண்டில், மாறிவரும் அரசியல் போக்குகளின் காரணமாக அறபு இலக்கியம் பெரும் செல்வாக்கைப் பெற்றது. அல்-நஹ்தா (மறுமலர்ச்சி) என்பது அரபு இலக்கியம் வளரத் தொடங்கிய காலம். 19 ஆம் நூற்றாண்டில் ஏற்பட்ட மாற்றங்களுக்கு அறபு இலக்கியம் மெதுவாக எதிர்வினையாற்றியது. மேற்கத்திய தொழில்நுட்பத்தின் முன்னேற்றத்திற்குப் பிறகு, மேற்கத்திய இலக்கியம் பிரபலமானது. எனவே, அறபு இலக்கியக் கவிதைகள் பல ஆண்டுகளாக மேற்கத்திய செல்வாக்கின் கீழ் வந்தன.' இந்த செல்வாக்கின் விளைவாக அறேபிய இலக்கியத்தில் 'மஹ்ஜர்' எனும் புலம் பெயர் இலக்கியம் வலுப்பெறத் தொடங்கியது (Osman \& Abdullah, 2017).

மேலே கூறப்பட்டுள்ள விடயங்களுடன் இருபதாம் நூற்றாண்டில் வாழ்ந்த லெபனானிய அறபு புலம்பெயர் கவிஞன் கலீல் ஜிப்ரான் (Kalil Gibran) இன் கவிதைகளை இவ்வாய்வு முதன்மைப்படுத்துகின்றது. அவரது கவிதைகளில் காணப்படும் தத்துவார்த்த உரையாடலில் காணப்படும் சமூகக் கருத்துக்களை வெளிக்கொணர்வதன் மூலம் கவிதை இலக்கியத்தின் மிக முக்கிய சமூகப் பணி குறித்து கருத்தாடல் மேற்கொள்ள முயற்சிக்கின்றது.

\section{ஆய்வு பிரச்சினை}

கவிதை என்பது ஒரு வகையான பொழுதுபோக்கு இலக்கிய வடிவம். இருப்பினும் அதனூடு தத்துவார்த்த ரீதியாக கருத்துக்கள் சமூகக் கருத்துக்களை இலகுவாக வெளிப்படுத்த முடியுமா என்ற வினா எழவாய்ப்புள்ளது. ஏனெனில் அது கற்பனை வழிவந்ததென்பதனால் உண்மைகளையும் உறுதியான விடயங்களையும் வெளிப்படுத்த முடியாது. அதேபோன்று இயந்திர வாழ்க்கையில் பல இன்னல்களுக்கும் பேரிடர்களுக்கும் உள்ளாகிய மனித குலம் கலை இலக்கியங்களை ரசிக்காமல் தம் வாழ்வை மட்டும் கவனித்துக் கொள்வதால் பல்வேறு உளவியல் பிரச்சினைகளுக்கு ஆளாகின்றனர். இலக்கியப் படைப்புகளைப் பொறுத்தவரை. சமூகப் பிரச்சனைகளைக் காண்பிப்பதிலும் அவற்றுக்கான 
தீர்வுகளை வழங்குவதிலும், நல்ல நடத்தை மற்றும் மதிப்புமிக்க கண்ணோட்டங்களைப் பரப்புவதிலும் குறிப்பிடத்தக்க முக்கியத்துவம் உண்டென்பது கட்டாயமாக வெளிப்படுத்தப்பட வேண்டியது.

\section{ஆய்வு நோக்கம்}

இவ்வாய்வு பின்வரும் நோக்கங்களைக் கொண்டுள்ளது:

1. கவிதை இலக்கியத்தையும், அறபுக் கவிதையின் சமூகப் பங்கினையும் அறிமுகம் செய்தல்.

2. லெபனானிய புலம்பெயர் கவிஞர் கலீல் ஜிப்ரானை அறிமுகம் செய்தல்.

3. கலீல் ஜிப்ரானின் கவிதைகளில் வெளிப்படுத்தப்படும் தத்துவார்த்த, சமூகக் கருத்துக்களை வெளிப்படுத்தல்.

\section{ஆய்வு முறையியல்}

ஆய்வு நோக்கங்களை அடைந்து கொள்வதற்காக விவரண மற்றும் பகுப்பாய்வியல் முறைகள் பயன்படுத்தப்பட்டன. முதலாம் நிலைத்தரவுகளுடன் இரண்டாம் நிலைத்தரவுகளாக நூல்கள், சஞ்சிகைள், பத்திரிகைகள் மற்றும் இணையம் போன்றவற்றின் மூலம் தரவுகள் பெறப்பட்டு ஆய்வுக்குட்படுத்தப்பட்டன.

\section{பெறுபேறுகளும் கலந்துரையாடலும்}

அறேபிய புலம்பெயர் (மஹ்ஜர்) இலக்கியம்

அறேபிய புலம்பெயர் இலக்கியம் என்பது, மஹ்ஜர் (مهر) இலக்கியம் என்று அழைக்கப்படுகிறது. இது ه எனும் வினையிலிருந்து தோற்றம் பெற்றது. 'தமது சொந்த (அறபு) நாடுகளிலிருந்து அரசியல், சமூக, பொருளாதார ரீதியில் முன்னேற்றமடைந்த நாடுகளுக்கு புலம்பெயர்ந்து சென்றுள்ள இலக்கியவாதிகளின் இலக்கியச் செயற்பாடுகளைக் இது குறிக்கிறது. குறிப்பாக, ஷhம் நாடுகளிலிருந்து (ஜோர்டான், லெபனான், பலஸ்தீன், சிரியா) வட, தென் அமேரிக்க நாடுகளுக்கு புலம்பெயர்ந்த அறேபியர் பல அறேபிய புலம்பெயர் சமூக மற்றும் இலக்கிய அமைப்புக்களை உருவாக்கி அதனூடாக பத்திரிகைகள், சஞ்சிகைளை வெளியிட்டனர். அவற்றில், கலீல் ஜிப்ரானின் தலைமையில் நியுயோர்க்கில் அமைந்த 'தாபிதத்துல் கலமிய்யா' முதன்மையானது' (Mohideen,2019).

அறேபிய இலக்கியத்தின் விருத்தியாகவே மஹ்ஜர் இலக்கியம் பார்க்கப்படுகின்றது. அதனூடு, பல்வேறு பிரச்சினைகள், வாழ்வியல் அனுபவங்கள், சமூக மாற்றங்கள் வெளிப்படுத்தப்படுகின்றன. பெரும்பாலும், சிறுகதை, நாவல், கவிதை, நாடகம் போன்றவை மஹ்ஜர் இலக்கியத்தின் வடிவங்களாக திகழ்கின்றன. பல்வேறு தலைப்புக்கள் பேசுபொருளாக உள்ளன. குறிப்பாக, புலம்பெயர் மக்கள் தமது சொந்த மண்ணிலும், குடியேறிய பகுதியிலும் தாம் எதிர்கொண்ட பிரச்சினைகள், அனுபவங்களை எழுதுகின்றனர். போர் ஏற்படுத்திய காணங்கள், தாயகத்தின் மீதான ஆகர்ஷpப்பும் காதலும் அதிகளவில் வெளிப்படுத்தப்படுகின்றன. இனத்துவ ரீதியில், புலம்பெயர் நாடுகளில் தாம் எதிர்கொள்ளும் ஒதுக்கலின் குரலாக மஹ்ஜர் இலக்கியம் ஒலிக்கிறது.

\section{தத்துவார்த்த உரையாடல் அறிமுகம்}

இலக்கியப் படைப்புக்களில் வாழ்வியல் குறித்த பார்வையை தர்க்கமாக வெளிப்படுத்துப்படுத்தக் கையாளும் உரையாடலை 'தத்துவார்த்த உரையாடல்' என சுருக்கமாக அறிமுகம் செய்யலாம். 'கி.மு. 19ஆம் நூற்றாண்டில் கிரேக்கத்தில் ஆரம்பமாகி, இன்று முதல் தத்துவத்துவார்த்த உரையாடல் இருந்து வருகின்றது. தத்துவார்த்த சிந்தனைக்கு உரையாடல் இன்றியமையாதது. இந்த வகையில், தத்துவார்த்த சிந்தனையானது, பொதுவான சிந்தனைசார் உரையாடலை முன்னெடுக்கின்ற சமூக நிபந்தனைகளில் தோன்றி வளர்கின்றது. தத்துவம் என்பது, உரையாடல்சார் சிந்தனை. அது அறிவை உருவாக்கும் வழிமுறை. ஆனால், இந்த நிபந்தனைகள் தத்துவத்துடன் மட்டுமே தொடர்புடையவையல்ல. மாறாக, தத்துவத்திற்கு அவசியமான அனைத்துப் பிரச்சினைகளையும் நோக்கும் ஒட்டுமொத்த சிந்தனை வடிவங்களுடனும் தொடர்புருகின்றது' （Wqydy, 1999). தத்துவார்த்த உரையாடல் வாழ்வுடன் 
பின்னிப்பிணைந்த ஒரு பகுதியாக உள்ளது. 'ஒரு நபரோ அல்லது ஒரு குழுவோ, தான் விரும்பியோ, விரும்பாமலோ உரையாடலை நாட வேண்டியுள்ளது. ஏனென்றால், மனிதனின் இயல்பும் அவனை இந்த உரையாடலை நாட வைக்கிறது, மேலும் பிரபஞ்ச நெறிகள் மற்றும் கடவுள் படைப்டைப் படைத்த வாழ்க்கை முறை ஆகியவை உரையாடலை ஒரு இயற்கை வாழ்க்கையை உருவாக்குவதற்கான நிபந்தனையாக ஆக்கியுள்ளன' (Al Sharood, 2019).

இதனால் தான் அது தொன்மையான வரலாற்றினையும் பாரிய சமூகப் பங்களிப்பினையும் கொண்டதாக உள்ளது. 'தத்துவ உரையாடல்கள் பண்டைய கிரேக்கத்தில் சமூக மரபுகளின் ஒரு பகுதியாக இருந்தன. சோக்ரடீஸ், பிளேட்டோ மற்றும் அரிஸ்டோட்டில் ஆகியோரின் பல போதனைகள், படைப்புகள் உரையாடல்களாக அல்லது கதைகளாக இருந்தன. இது தத்துவத்தின் வாயிலாக வந்த புரிதல்கள் மற்றும் கருத்துக்கள் அன்றாட வாழ்வில் பயன்படுத்தப்பட வேண்டியவை' (Triliva \& Dafermos, 2007). 'ஒரு நடைமுறை தத்துவவாதி' (Practicing Philosopher) தன் வாடிக்கையாளருடன் வாழ்க்கை கொண்டு வரும் கேள்விகளை தத்துவமாக்குகிறார். தத்துவஞானி தத்துவ அ அறிவயும் அனுபவத்தையும் ஒரு சாதாரண மனிதனுக்கு (தத்துவவாதி அல்லாதவனுக்கு) தனது சொந்த தத்துவம், வாழ்க்கை முறையைக் கட்டமைத்து, அதைப் பயன்படுத்த உதவுவதற்காக முயல்கிறார். பழங்காலத்திலிருந்தே நெய்யும் நூல் போன்ற தத்துவ பாரம்பரியத்தை அடிப்படையாகக் கொண்ட வாழ்க்கையின் கருத்தாக்கமாக தத்துவத்தை தத்துவ ஆலோசனை முன்வைக்கிறது. தத்துவ ஆலோசனையானது விமர்சன சிந்தனையை அடிப்படையாகக் கொண்டது. கேள்விகளை முன்வைக்கும் சோக்ரடிக் முறையைப் பயன்படுத்துகிறது. இது ஒரு உரையாடல் செயல்முறையாகும். இது இயங்கியல் சிந்தனையைக் கட்டுப்படுத்துவதுடன், அன்றாட வாழ்க்கையில் எழும் அச்சங்கள் மற்றும் கேள்விகள் மற்றும் ஒட்டுமொத்த வாழ்க்கையின் அர்த்தம் பற்றிய கேள்விகளையும் பிரதிபலிக்கிறது' (Sulavikova, 2011).

\section{ஜிப்ரான் கலீல் ஜிப்ரான்}

ஜிப்ரான் கலீல் ஜிப்ரான் ஏழைக் குடும்பமொன்றில் பிறந்து வளர்ந்த லெபனானியக் கவிஞர். அறேபிய கவிதையின் பாரம்பரிய மரபுகளில் தேர்ச்சியுள்ளவர். அவரது சிந்தனைகள் தத்துவார்த்தப்பார்வை கொண்டவையாக இருந்தன. அவருடைய இளம் வயதில் அமெரிக்காவின் பாஸ்டன் நகருக்கு அவர் குடும்பம் இடம் பெயர்கிறது. மிகுந்த வறுமையில் வாடிக் கொண்டிருந்த சிறுவனான கலீல் ஜிப்ரான், ஓவியம் வரைவதில் ஆர்வம் உடையவராக இருந்தார். ஓவியக் கண்காட்சி நடத்துகிறார். கவிதை, சிறுகதை எழுதுவதிலும் நாட்டம் கொண்டார்.

'ஜிப்ரான் இன்றைக்கு லெபனானில் 1883 ஜனவரி 6ஆம் தேதி பிறந்தார். தன் தாய் கமிலாவிற்கு 30 வயதாக இருக்கும் போது தாயின் மூன்றாவது கணவனான கலீல் என்பானுக்குப் பிறந்தார். தாத்தா ஒரு பாதிரியார். தனது குடும்பத்தின் ஏழ்மையினால் சிறுவர் பருவத்தில் கல்வியைப் பெறமுடியாமல் போன ஜிப்ரான் தனது வட்டிற்கு வருகை தரும் பாதிரியாரான தாத்தா மூலம் பைபிளையும், அராபிக், சிரியாக் ஆகிய மொழிகளையும் கற்றுக்கொண்டார். ஜிப்ரானுக்கு எட்டு வயது வாக்கில் அவருடைய தந்தை ஊழல் குற்றத்திற்கு உள்ளாகி சிறையில் அடைக்கப்பட்டது மட்டுமல்லாமல், அவருடைய குடும்பச் சொத்துக்களும் அதிகாரிகளால் பறிமுதல் செய்யப்பட்டன. இத்தகைய கையறு நிலையில் குடும்பம் 1895-இல் அமெரிக்காவிற்குப் புலம்பெயர்ந்தது. அமெரிக்காவில் அவருடைய தாய், வீடு வடாகச் சென்று தைக்கிற தையற்காரியாகவும், நாடா, துணி மணி விற்பவராகவும் உழைத்துக் குடும்பத்தைக் காப்பாற்றியுள்ளார். அமெரிக்காவில்தான் ஜிப்ரான் 1895-லிருந்து முறையாகப் பள்ளிக்கூடம் ேோகத் தொடங்கியுள்ளார். கூடவே ஒரு கலைப் பள்ளியிலும் சேர்ந்து பயின்றுள்ளார். இந்நிலையில் மேற்குலக அழகியல் பண்பாட்டைவிடத் தன் மண்ணின் பண்பாட்டையும் மரபையும் உள்வாங்க வேண்டும் என்ற நோக்கில் அவருடைய தாய் மீண்டும் ஜிப்ரானை லெபனானுக்கே திருப்பி அனுப்பிவிட்டார். அப்பொழுது அவருக்கு வயது 15. பல ஆண்டுகள் கழித்து உயர்கல்வியை பெய்ரூட்டில் முடித்த பிறகு 1902-இல் மீண்டும் அமெரிக்காவிற்குத் திரும்பினார். அவர் திரும்புவதற்கு இரண்டு வாரத்திற்கு முன்புதான் அவருடைய சகோதரி சுல்தானா காசநோயினால் தனது 14 வயதில் இறந்திருந்தார். ஜிப்ரான் அமெரிக்காவிற்கு வந்த அடுத்த ஆண்டில் அவருடைய சகோதரர் பீட்டர் அதே நோயினால் இறந்தார். தாய் புற்றுநோயினால் இறந்தார். சகோதரி மரியன்னாதான் ஜிப்ரானைக் கவனித்துக் கொண்டார். அவள் ஓர் ஆடை அலங்காரக் கடையில் பணியாற்றினாள். கலை மனம் மிக்க 
ஜிப்ரானுக்குள் இத்தகைய வாழ்க்கை கொடுத்த துக்கம்தான் அவர் எழுத்துக்களுக்குள் துன்பமாகவும் தத்துவமாகவும் கேலியும் கிண்டலுமாகவும், விளையாட்டாகவும் விரவிக் கிடக்கின்றன. 1923இல் ஆங்கிலத்தில் எழுதிய தீர்க்கதரிசி (The Prophet) என்ற நூல் அவருக்கு உலகப் புகழ் வாங்கித்தந்தது. புலம்பெயர்ந்த நியூயார்க் நகரத்தில் 1931ஆம் ஆண்டு ஏப்ரல் 10 ஆம் தேதி தனது 48வது வயதில் எலும்புருக்கி நோயினால் இறந்தார்' (Panjangam, 2014).

\section{கலீல் ஜிப்ரானின் கவிதைகளில் வெளிப்படும் தத்துவார்த்த உரையாடல்கள்}

இந்த வகைக்குள் கலீல் ஜிப்ரானின் 'அல்-மவாகிப்' எனும் கவிதைக் தொகுதி உள்ளடங்களின்றது. அது நீண்டதொரு தொகுதி. மொத்தமாக 203 மரபுசார் பாடல்களைக் கொண்டது. ஜிப்ரான் தனது கருத்துக்களை வெளிப்படுத்த தத்துவார்த்த உரையாடல் முறையனை இக்கவிதைகளில் பின்பற்றுகின்றார்.

இக்கவிதைகளில், ஜிப்ரான் வாழ்வில் ஏற்படும் நல்லது, கெட்டது குறித்து பேச ஆரம்பிக்கிறார். மனிதர்கள் தீமையில் நாட்டம் கொண்டவர்களேயன்றி நல்லதை செய்யமாட்டார்கள். அப்படிச் செய்தாலும் அது காலத்தின் கட்டாயமாகத்தான் இருக்கும் என்கிறார். பலவான்கள் பலவீனர்களின் உள்ளங்களை 'கருவிகள்' போல் மாற்றயமைக்கும் காலத்தில் வாழ்வதாக வர்ணிக்கின்றார். ஆனால், மனிதர்கள் மாறும் காலம் வரும். அப் போது இந்த தீர்மானிக்கும் வகையினர் மாண்டு போவர் என்கிற வாழ்வியல் யதார்த்தத்தைப் பாடுகிறார். மானிதர்கள் மந்தைக் கூட்டத்தின் பின்னால் போகும் இடையனைப் போல எவ்வித இலட்சியமுமற்று வாழ்கின்றனர் எனச் சாடுகின்றார். இதுவே மனித குலத்தின் அழிவுக்குக் காரணமென்கிறார்.

எடுத்துக்காட்டு: 1

$$
\begin{aligned}
& \text { جُبروا إذا مصنوعٌ الناس في الخير }
\end{aligned}
$$

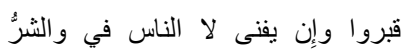

$$
\begin{aligned}
& \text { تحركها آلاتّ الناس وأكثر } \\
& \text { تنكسرُ ثم يوماً الدهر أصابع } \\
& \text { علمّ عالم هذا تقولنَّ فلا }
\end{aligned}
$$

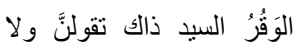

$$
\begin{aligned}
& \text { بها يسير قطعانّ الناس فأفضل }
\end{aligned}
$$

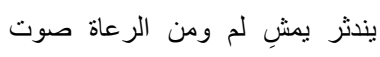

$$
\begin{aligned}
& \text { கட்டாயப்படுத்தப்பாட்டால்தான் மனிதர் நல்லது செய்வர் } \\
& \text { மண்ணறை செல்லுமட்டும் அவர்களில் தீமையழியாது }
\end{aligned}
$$$$
\text { காலத்தின் விரல்கள் அசைக்கும் கருவிகளே அதிகமாவோர் }
$$

பின்னர், ஒருநாள் அவை உடைந்துவிடும்

அது அறிவுடைய உலகமென்றோ,

அங்கு சங்கையான தலைவருண்டென்றோ கூறாதே!

மனிதர்களிலும், இடையனின் சத்தம் கொண்டுசெல்லும் மந்தைகள் சிறந்தவை.

அவ்வாறு நடக்காதவன் அழிந்து விடுவான்!

எடுத்துக்காட்டு: 2

வனங்களில் சட்டங்கள் உள்ளிட்ட அனைத்து விஷயங்களும் மறைந்தே கிடக்கும். ஏனெனில், அனைத்துப் பொருட்களை அதன் நிறம், தோற்றம் தாண்டி வனம் ஏற்கிறது. அங்கு, சமயமோ அல்லது நிராகரிப்போ எதவுமில்லை. இக்கருத்தை மிக அழகாக கவிஞர் இப்படிப் பாடுகிறார்.

$$
\begin{aligned}
& \text { القطيخ فيها ولا لا راعِ الغابات في ليس }
\end{aligned}
$$

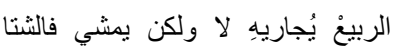




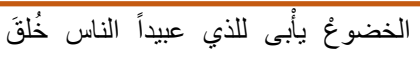

الجميغ سار سائراً يوماً هبَّ ما فإذاذا لإنا

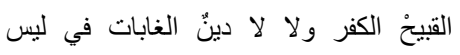

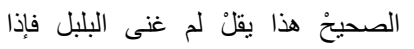

வனங்களில் இடையனில்லை மந்தைகளுமில்லை

குளிர் காலம் செல்கிறது ஆனாலும் அதை வசந்த காலம் தொடராது

அடிபணிய மறுப்பவனுக்கு மனிதர்கள் அடிமைகளாக படைக்கப்பட்டுள்ளனர்

ஓருநாள் வரும்போது அனைவரும் சென்று விடுவர்

வனங்களில் சமயமோ அதை நிராகரித்தலோ இல்லை

குயில் இசைத்தால் இது சரியென்று கூறுவதில்லை.

எடுத்துக்காட்டு: 3

நீதி குறித்து இப்படிப் பாடுகிறார்:

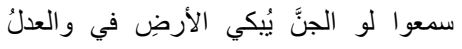

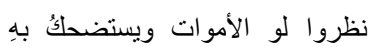

$$
\begin{aligned}
& \text { صغروا إن للجانين والموتُ فالسجنُ }
\end{aligned}
$$

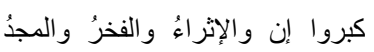

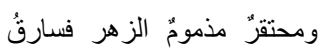

$$
\begin{aligned}
& \text { الخطر الباسلُ يُدعى الحقل وسارق الزهر }
\end{aligned}
$$

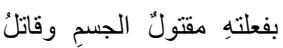

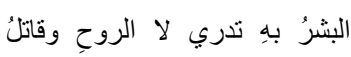

பூமியிலுள்ள நீதி பற்றி கேள்விப்பட்டால் ஜின்களும் சிரிக்கும்

மரணித்தவர்கள் பார்த்தால் சிரிப்பார்கள்

சிறையும் மரணமும் அற்பர்களாக குற்றவாளிகளுக்கே

கீர்த்தியும், பெருமையும், சிறப்பும் பெரும் புள்ளிகளுக்கே

மலரைப் பறித்தவன் கேவலமான திருடனாம்

வயல் காணியைப் பறித்தவன் வீரனமாம்

உடலைக் கொன்றவன் கொலை செய்யப்படுகிறான்

ஆன்மாவைக் கொன்றவன் பற்றி மனிதருக்குத் தெரியாது.

சமூகத்தில் காணப்படும் நீதியை கிண்டலடிக்கிறார். الجن (ஜின்கள் சிரிக்கும்) என்ற வார்த்தை எந்தளவு மனிதர்களுக்கிடையில் நீதி மறைந்து அநீதி தலைவிரித்தாடுகிறது என்பதை

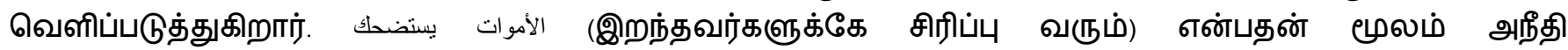
தலைவிரித்தாடும் இந்த உலகில் வாழும் மனிதர்களின் இயலாமையையும், கையாலாகாத் தனத்தையும் குறிப்பிடுகிறார். அத்துடன், மனிதன் நீதி என்ற பெயரில் அநீதியையே செய்கிறான். பலசாலிக்கு ஒரு நீதி, பலவீனனுக்கு ஒரு நீதி என்று தீர்ப்புக்கள் வேறுபடுவதாக சினம் கொள்கிறார்.

எடுத்துக்காட்டு: 4

கல்வி பற்றிய ஜிப்ரானின் தத்துவார்த்த உரையாடல் இவ்வாறு செல்கிறது:

أوَّلها بأنَ سبلٌ الناس في والعلُّ

والقدرُ فالدهر أواخرها أما فالمان

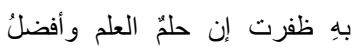

سخروا الكرى أبناء بين ما وسرتَ 
منفرداً الأحلام أخا رأيتَ فإن

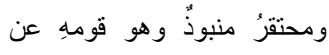

மனித அறிவின் ஆரம்பம் சில வழிகள்

அதன் முடிவு காலமும் விதியும்

வென்றால் கனவு பாண்பதே அறிவில் சிறந்தது

தூக்கத்தில் மிதப்பவர்களுக்கு மத்தியில் நீ சென்றால் கேலிசெய்வர்

கனவு காண்பவனை சமூகத்தை விட்டு ஒதுங்கியிருப்பதைப் பார்த்தால் அவன்

கேவலாமானவன், புறக்கணிக்கப்பட்டவன்

அறிவின் ஆரம்பம் அறிதல், முடிவு மனித மரணம்தான் என்கிறார். சமூகத்தை விட்டு ஒதுங்கிய இருப்பதற்கு நிகராக கற்றலில் ஈடுபடல் வேண்டும் என்கிறார். சமூகத்தின் எளனம், பரிகசிப்பை பொருட்படுத்தக் கூடாதென்கிறார். கற்பவன் கடுமையான முயற்சியில் இருக்க வேண்டும் என்பது ஜிப்ரானின் அழைப்பாகும்.

எடுத்துக்காட்டு: 5

மகிழ்ச்சி என்பது நடக்காத ஒரு யூகம் என்கிறார் ஜிப்ரான். அது நடந்தால் மனிதன் சடைவடைந்து விடுவான். மனிதன் ஆசைகளை அடைவதில்தான் மகிழ்ச்சி இருப்பதாக நம்புகிறான் என்று விமர்சிக்கிறார்.

$$
\begin{aligned}
& \text { شُبح سوى الدنيا في السعادة وما }
\end{aligned}
$$

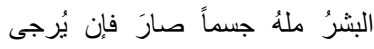

$$
\begin{aligned}
& \text { مكتهاً السهل نحو يركض كالنهر }
\end{aligned}
$$

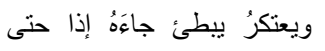

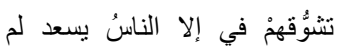

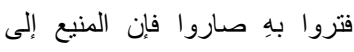

$$
\begin{aligned}
& \text { منصرفت وهو سعيداً لقيتَ فابن الن } \\
& \text { العبرُ خُلقهِ في فقل المنيع عن العن } \\
& \text { மகிழ்ச்சி என்பது விரும்பும் பேய்தான். } \\
& \text { அது உடலானால் மனிதர் சடைவடைவர். } \\
& \text { சமவெளியை நோக்கி திரண்டோடி வந்து } \\
& \text { வேகம் குறைந்தும் தேங்கி நிற்கும் நதி போல } \\
& \text { நடக்காததற்காக மக்கள் தங்கள் ஏக்கத்தில் மட்டுமே } \\
& \text { மகிழ்ச்சியாக இருந்தனர். } \\
& \text { நடக்காததை விட்டு விலகி மகிழ்ச்சியாக இருப்பவரை நீ கண்டால் } \\
& \text { அவரது நடத்தையில் படிப்பினை இருப்பதாகச் சொல். }
\end{aligned}
$$

\section{முடிவுரை}

ஜிப்ரான் கலீல் ஜிப்ரான் இக்கவிதைகளில் தனது தத்துவ சிந்தனைகள் மற்றும் சமூகப் பார்வைகள் அனைத்தையும் எழுதி, இயற்கையின் உணர்வுகளுடனும் அதன் விவரங்களுடனும் அதில் உரையாடி, இயற்கையே முழுமையான மகிழ்ச்சிக்குக் காரணம் என்றும், போலித்தனத்திலிருந்து விலகி உலகமே என்றும் வாதிடுகிறார். இதுதான், சடவாதத்தின் பொய்யான உலகத்திலிருந்து விலகி பரிபூரணமான மனித வாழ்க்கைக்குப் பின்னால் தங்கள் தொலைதூர இலக்கை அடைய பாடுபடும் ஆத்மாக்களின் பண்பு என வர்ணிக்கிறார். இலக்கியங்கள் கால நிகழ்வுகளை எடுத்துரைக்கும் சான்றுகளாகும். அவற்றை இயற்றும் எழுத்தாளர்கள் அ அக்காலத்தின் பிரதிநிதிகளாவர். சிறந்த 
படைப்பாளிகள் என்று கருதப்பட்டவர்கள் இயற்றிய படைப்புக்களில் காலத்தை வென்று விடவில்லை.' (கயல்விழி, 2021, பக்: 1) ஆயினும், கலீல் ஜிப்ரான் தன்னை காலம் முழுவதும் பேசும்படி செய்து விட்டார். அறேபிய புலம்பெயர் கவிதைகள் அறபு இலக்கியத்தின் மிகப்பலமானதொரு விடிவம் என்ற வகையில் இது தொடர்பான பரந்த ஆய்வுடன் கூடிய விழிப்புணர்வினை அறபு மொழி கற்பிக்கும் நிறுவனங்கள், கல்விக் கூடங்கள் எதிர்காலத்தில் மாணவர் மத்தியில் ஏற்படுத்தி திறனாய்வினை வலுவூட்டும் கலைத்திட்டங்களை உருவாக்க வேண்டும் என்றும் இது தொடர்பான வழிகாட்டல் மற்றும் பயிற்சிகளை வழங்க வேண்டுமென்றும் இவ்வாய்வு பிரேரிக்கின்றது.

\section{References}

Al Sharoud, Ali Jabir Alabd, (2019) Alhiwar mafhuman watasilan wawaqiean, Yearbook of the Faculty of Islamic and Arabic Studies for Girls in Alexandria, 2(35).

As'seyyat, A.H, (n.d) Tarikul atapil arapi, Tarunnahla misr, Cairo.

Maunaguru, C., Chirtalega, MAU., \& Nuhman, MA., (1979) Twentieth Century Ceylon Tamil Literature, Readers' Association, Kalmunai, Sri Lanka.

Mohideen, HLM., (2019) Optimistic Perceptions in Arabic Diaspora Literature - A Special Reference With Elia Abu Madi's Poems, Sri Lankan Journal of Arabic and Islamic Studies, 2(2), 10-19.

Osman, R., \& Abdullah, N., (2017) Development of Islamic values through Arabic poetry; Contribution of Arabic literature websites, Al-Shajarah, 22, 151-174.

Panjangam, (2014) Tunpankalaip pataippakkat terintavar, Keetru Online journal, https://www.keetru.com/index.php/2014-03-08-04-35-27/2014-03-08-12-18-14/26952-2014-08-08-11-00$\underline{44}$

Samar Sadr. (2021) Tahlil qasidat almawakib, Baytalqaseed Online journal.

Sulavikova, B., (2011) Philosophical dialogue as a space for seeking a good life, identity and critical thinking, Slovakia: Institute for Research in Social Communication, Human Affairs, 21, 157-162. https://doi.org/10.2478/s13374-011-0017-5

Triliva, S., \& Dafermos, M., (2007) Philosophical Dialogues as path to a more Positive Psychology, Journal of Community \& Applied Social Psychology, 18, 17-38.

Wqydy, M., (1999) Jur'at almawqif alfalsafii, Afrikia Shark, Morocco.

\section{Funding}

No funding was received for conducting this study.

\section{Conflict of interest}

The Author has no conflicts of interest to declare that they are relevant to the content of this article.

\section{About the License}

(C) The Author 2022. The text of this article is open access and licensed under a Creative Commons Attribution 4.0 International License

\section{Cite this Article}

MHA. Munas, Philosophical dialogue in Arabic diaspora literature with special reference to Kahlil Gibran's poems, Indian Journal of Tamil, 3(1) (2022) 13-20. https://doi.org/10.54392/ijot2213 